Jenny Fernanda Alvarez-Matute; Darwin Gabriel Garcia-Herrera; Cristián Andrés Erazo-Álvarez; Juan Carlos Erazo-Álvarez

http://dx.doi.org/10.35381/e.k.v3i6.827

\title{
GeoGebra como estrategia de enseñanza de la Matemática
}

GeoGebra as a Mathematics teaching strategy

\author{
Jenny Fernanda Alvarez-Matute \\ jfalvarezm@ucacue.edu.ec \\ Universidad Católica de Cuenca, Azogues \\ Ecuador \\ https://orcid.org/0000-0003-1990-9719 \\ Darwin Gabriel Garcia-Herrera \\ dggarciah@ucacue.edu.ec \\ Universidad Católica de Cuenca, Azogues \\ Ecuador \\ https://orcid.org/0000-0001-6813-8100 \\ Cristián Andrés Erazo-Álvarez \\ cristianerazo@ucacue.edu.ec \\ Universidad Católica de Cuenca, Cuenca \\ Ecuador \\ https://orcid.org/0000-0001-8746-4788 \\ Juan Carlos Erazo-Álvarez \\ jcerazo@ucacue.edu.ec \\ Universidad Católica de Cuenca, Cuenca \\ Ecuador \\ https://orcid.org/0000-0001-6480-2270
}

Recepción: 09 abril 2020

Revisado: 15 de mayo 2020

Aprobación: 15 junio 2020

Publicación: 1 de julio 2020 


\title{
RESUMEN
}

El objetivo estuvo centrado en describir el uso de la herramienta GeoGebra como alternativa en el proceso de enseñanza-aprendizaje de la Matemática, a fin de proponer una guia para la formulación de un plan de estrategia didáctica dirigido a los docentes del área de matemática de la ciudad de Azogues. Se fundamentó desde el enfoque positivista y se desarrolló con base en la metodología cuantitativa. La población estuvo conformada por 84 docentes del área de matemática. Los resultados de la tabla 2 revelan que el $71,8 \%$ de los docentes encuestados ocasionalmente, casi nunca y/o nunca utilizan algún tipo de software educativo para impartir su cátedra de Matemática. EI modelo ADDIE propuesto para la implementación del uso de GeoGebra en el aula permite al docente identificar los aspectos positivos y negativos en cada fase del ciclo metodológico, para mejorar y renovar sus actividades didacticas y así obtener una mejor participación estudiantil.

Descriptores: Informática educativa; programa informático didáctico; tecnologíaeducacional; álgebra. (Palabras tomadas del Tesauro UNESCO).

\begin{abstract}
The objective was focused on describing the use of the GeoGebra tool as an alternative in the teaching-learning process of Mathematics, in order to propose a guide for the formulation of a didactic strategy plan aimed at teachers of the mathematics area of the city of Azogues. It was based on the positivist approach and was developed based on quantitative methodology. The population was made up of 84 teachers in the area of mathematics. The results of Table 2 reveal that $71.8 \%$ of the teachers surveyed occasionally, almost never and / or never use some type of educational software to teach their mathematics chair. The ADDIE model proposed for the implementation of the use of GeoGebra in the classroom allows the teacher to identify the positive and negative aspects in each phase of the methodological cycle, to improve and renew their didactic activities and thus obtain better student participation.
\end{abstract}

Descriptors: Computer uses in education; educational software; educational technology; algebra. (Words taken from the UNESCO Thesaurus). 
Jenny Fernanda Alvarez-Matute; Darwin Gabriel Garcia-Herrera; Cristián Andrés Erazo-Álvarez; Juan Carlos Erazo-Álvarez

\section{INTRODUCCIÓN}

Desde tiempos remotos la matemática ha sido considerada como un aprendizaje básico para el desarrollo del pensamiento dentro de todos los sistemas formales de educación. Es por ello que el (Ministerio de Educación del Ecuador [MINEDUC], 2016) en el marco curricular plantea que el proposito de la enseñanza de la matematica gira en relación a que los estudiantes sean capaces de razonar, pensar, relacionar y aplicar los conocimientos y premisas matemáticas a situaciones reales de la vida cotidiana. No obstante, este propósito conlleva a considerar el aprendizaje de la matemática como un proceso muchas veces difícil, dada la complejidad, la exactitud y lo abstracto que resultan muchos contenidos para los estudiantes, más en un contexto pedagógicodidáctico que puede resultar deficiente respecto al desempeño estratégico del docente. En este sentido (Orrantia, 2006) señala que es una inquietud perenne de los profesionales dedicados a la docencia entender los problemas que se generan en torno a la enseñanza y el aprendizaje de la Matemática, considerando los altos índices de fracaso que muestran los estudiantes en esta disciplina, en todos los niveles educativos. Tal es el caso de las dificultades que representa comprender y graficar conceptos y problemas matemáticos, sea en la pizarra o en el cuaderno; tanto para el docente, que muchas veces no posee las estrategias y herramientas adecuada, como para el estudiante que le resulta difícil por posibles problemas de comprensión y hasta tedioso por no sentirse motivado hacia este tipo de aprendizajes.

Poniendo en contexto lo planteado, los problemas y dificultades que se presentan en el Ecuador y que atañen al aprendizaje de la matematica son bastante significativos, evidencia de ello esta contenida en los resultados obtenidos en la evaluacion educativa para el periodo 2017-2018, reallizada por el Instituto Nacional de Evaluación Educativa (INEVAL, 2018). En este sentido, la prueba nacional Ser Estudiante refleja que el 53.3\% de la muestra de estudiantes de $4^{\circ}, 7^{\circ}$ y $10^{\circ}$ de Educación General Básica (EGB) muestran un nivel de insuficiencia en el campo de la Matemática. Asimismo, los 
resultados de la prueba Ser Bachiller, revelan que este campo de la Matemática tiene el nivel de logro de insuficiente más alto y el cual está representando por el $27,5 \%$ de la muestra de estudiantes de $3^{\circ}$ de Bachillerato General Unificado (BGU).

Estos niveles son indicadores en cuanto a la realidad del aprendizaje y los conocimientos adquiridos en la matemática por parte del estudiante ecuatoriano dentro del proceso educativo y que dan a entender que los conocimientos no son asimilados de manera idonea, desencadenando desisteres y desmotivacion en ellos. Por esto, el Sistema Educativo debe abocarse al desarrollo de acciones concretas que coadyuven al mejoramiento de la enseñanza-aprendizaje de la Matemática.

Significa, también, que el docente debe afrontar esta situación, que por demás es muy preocupante, con la busqueda y aplicación de metodos y estrategias pertinentes para que el proceso didáctico de la Matemática sea más dinámico, interesante, y que haga que el estudiante se convierta en un sujeto activo en la gestión de su propio proceso de aprendizaje. Planteamiento que es convalidado por (Rodríguez-Uribe, 2017) cuando afirma que la enseñanza de la Matemática se ha convertido en un desafío para la mayoría de los educadores, quienes deberían tratar de incorporar nuevas formas de enseñar y llegar a los educandos a través del uso de estrategias y recursos innovadores, dejando de lado lo tradicional e incorporando nuevas formas de enseñar.

Entonces, como posibilidad para mejorar el proceso didáctico en el área de la Matemática, los sistemas educativos han comenzado a incorporar formalmente estrategias que promueven la innovación e integración de recursos tecnológicos; dado que, en la actualidad la tecnología está inmersa en todos los aspectos de la vida cotidiana de las personas y cada vez son mayores los beneficios que esta ofrece a la sociedad.

Significa entonces, que los docentes recurren cada vez más al uso de las Tecnologías de la Información y la Comunicación (TIC) para desarrollar el proceso de enseñanzaaprendizaje mediante herramientas como las plataformas y aplicaciones educativas, lo 
Jenny Fernanda Alvarez-Matute; Darwin Gabriel Garcia-Herrera; Cristián Andrés Erazo-Álvarez; Juan Carlos Erazo-Álvarez

que ha generando que los estudiantes y los propios docentes desarrollen habilidades y destrezas de mejor manera, contribuyendo significativamente con la calidad educativa (Arévalo-Duarte \& Gamboa-Suárez, 2015).

Cabe agregar que el manejo adecuado de las TIC en el aula ayuda a la participación activa de los estudiantes, ya que se está en una época en la que ellos, como nativos digitales, están inmersos en un mundo donde la mayoría de las actividades que realizan día a día están dominadas por el uso de diversos dispositivos y programas electrónicos. En atención a esto, entonces, es importante que el docente cuente con los recursos necesarios y la preparación oportuna en cuanto al manejo de estrategias y tecnología para el aprendizaje mediado por las TIC (Erazo-Álvarez \& Narváez-Zurita, 2020).

En lo que refiere a las plataformas y aplicaciones educativas, es de saber que este tiene la finalidad de captar la atención de los estudiantes y lograr cambios sustanciales en su aprendizaje. Pues como expresa (Saltos-Cedeño, et al., 2020) el uso de TIC en el aula y específicamente en el área de matemáticas promueve un aprendizaje significativo y no mecanicista, dado que se relacionan los nuevos conocimientos con los existentes, además ayuda al estudiante a desarrollar el razonamiento lógico y matemático.

Asimismo, estas plataformas y aplicaciones educativas son herramientas de tipo software informático que sirve como soporte para la adquisición de conocimientos, que transforma el rol del estudiante haciéndolo más activo y participativo, descentrando al docente como eje del proceso (Fernández, et al., 2017). Es por ello que para mejorar el nivel de conocimiento de los estudiantes en las diferentes áreas y sobre todo en las matemáticas se deben incorporar estos tipos de recursos educativos innovadores que permiten generar cambios significativos en la forma de enseñar y aprender. Asi, tanto docentes como estudiantes, no se ven limitados al uso de materiales convencionales; sino, más bien opten por la utilización de programas o aplicaciones focalizadas en la interacción, el trabajo activo y cooperativo y la autogestion del aprendizaje por parte del 
estudiante (Rodríguez, Erazo, \& Narváez, 2019).

En la actualidad existen un sin número de plataformas y aplicaciones educativas para el abordaje de la Matemática que permiten realizar un trabajo educativo más eficaz, haciendo que el estudiante no esté preocupado en repetir constantemente ejercicios de manera abstracta y mecánica, por el contrario, al mediar el proceso con estas herramientas, el trabajo de aprendizaje se centra en analizar y comprender de manera detallada cómo se desarrollan los procesos matemáticos.

En este tenor, una de las aplicaciones educativas más completas es sin duda alguna GeoGebra. Al respecto, afirma (Pari-Condori, 2019) que GeoGebra es un software de gran ayuda para la enseñanza de la Matemática, al que se puede acceder libremente ya sea de manera online $u$ offline, que soporta múltiples plataformas y engloba geometría, álgebra, aritmética, análisis, estadística y probabilidades en un solo programa. Lo que quiere decir que es una aplicación completa que involucra todas las ramas de la matemática, tiene una interface interactiva, sencilla y amigable que permite comprender de mejor manera los conceptos matemáticos para enriquecer el conocimiento y obtener un aprendizaje significativo.

El uso de la plataforma GeoGebra en las clases de Matemática supone un cambio significativo, pues favorece a los esudiantes en cuanto al entendimiento de forma más eficiente mediante gráficos, demostraciones y simulaciones matemáticas. Cabe resaltar, que esta plataforma ha generado una gran demanda sobre todo en el nivel superior, siendo necesario sentar bases sólidas para que los estudiantes aprendan y desarrollen su pensamiento lógico matemático mediado por el GeoGebra desde los niveles básicos. A razón de esto son muchos los autores, tanto del ambito Latinoamrericano como del propio Ecuador, que han aportando ideas y plantemientos en relación al GeoGebra como herramienta que favorece la adquisición de conocimientos y promueve mejoras en el rendimiento académico y la motivación de los estudiantes en las diferentes ramas de estudio de la Matemática. En este tenor, (Salas-Rueda, 2018) con su estudio 
Jenny Fernanda Alvarez-Matute; Darwin Gabriel Garcia-Herrera; Cristián Andrés Erazo-Álvarez; Juan Carlos Erazo-Álvarez

realizado en México sobre el impacto que tiene la plataforma de GeoGebra en la nube, expresa que es una herramienta indispensable para la enseñanza-aprendizaje de la matemática; dado que es un programa de fácil manejo, que permite graficar todo tipo de funciones, mejora notablemente el rendimiento académico de los estudiantes al afianzar sus conocimientos en relación a la geometría, el álgebra, el cálculo y la estadística.

Asimismo, (Vargas-Vargas \& Gamboa-Araya, 2013) desde su experiencia investigativa realizada en Costa Rica con estudiantes de nivel secundario refieren que al realizar actividades de aprendizaje sobre el Teorema de Pitágoras con el programa GeoGebra, estos se vieron motivados hacia el aprendizaje, mejorando con ello sus conocimientos y la confianza hacia sí mismo. Al indagar sobre la percepción que tienen los maestros sobre las ventajas del GeoGebra para el aprendizaje de la Matemática desde el contexto de la escuela colombiana, (Tamayo-Martínez, 2013) enuncian que esta es una herramienta que permite la interacción dinámica entre el estudiante y la Matematica; ademas, es una instrumento util para indagar en los conocimientios previos y afianzar la cosntruccion de nuevos conocimientos al permitirle a los estudiantes explorar los contenidos con mayor precisión.

Situándonos en el contexto ecuatoriano, se tiene que (Barahona-Avecilla, et al., 2015) con su investigación sobre la influencia del programa GeoGebra en el aprendizaje de las ciencias exactas realizado en la Escuela Superior Politécnica de Chimborazo, exponen que la correcta utilización del programa GeoGebra resulta beneficioso para alcanzar un mejor nivel de conocimiento en los estudiantes y por lo tanto un rendimiento académico favorable. De la misma manera, (Guerrero-Garcés, et al., 2018) con su investigación exponen que cuando los docentes emplean la herramienta GeoGebra como recurso didáctico para el razonamiento, comprensión y resolución de problemas de inecuaciones, esto incide de manera satisfactoria en el mejoramiento del rendimiento académico de los estudiantes universitarios. 
Jenny Fernanda Alvarez-Matute; Darwin Gabriel Garcia-Herrera; Cristián Andrés Erazo-Álvarez; Juan Carlos Erazo-Álvarez

Cabe decir que la plataforma GeoGebra permite enriquecer los conocimientos de los estudiantes, en el sentido de que estos pueden comprender mejor los conceptos matemáticos a través de demostraciones y simulaciones gráficas, en la pantalla del computador o celular (Cordero-Naspud, et al., 2020); lo que brinda múltiples beneficios, ya que es un software accesible de manera online que puede ser utilizado en cualquier lugar y en diferentes dispositivos como computadoras, smartphone y tablet (Carrillo de Albornoz-Torres, 2019).

Todo lo planteado evidencia que la plataforma tecnológica GeoGebra es una herramienta educativa que se presenta como propuesta didáctica favorable para el proceso de enseñanza-aprendizaje de la Matemática, pues el docente cambia significativamente la forma de enseñar y el estudiante se convierte en sujeto que lideriza su autoformación para una mejor comprensión de los procesos matemáticos. En concreto, se puede decir que esta herramienta otorga ventajas significativas en cuanto a la transformación del proceso de enseñanza, la construcción de conocimientos, el rendimiento académico y la motivación de los estudiantes hacia la Matemática.

Finalmente, lo enunciado motivó el desarrollo de la presente investigación, cuyo objetivo estuvo centrado en describir el uso de la herramienta GeoGebra como alternativa en el proceso de enseñanza-aprendizaje de la Matemática, a fin de proponer una guia para la formulación de un plan de estrategia didáctica dirigido a los docentes del área de matemática de la ciudad de Azogues. 
Jenny Fernanda Alvarez-Matute; Darwin Gabriel Garcia-Herrera; Cristián Andrés Erazo-Álvarez; Juan Carlos Erazo-Álvarez

\section{MÉTODO}

Considerando que con el estudio se buscó describir el uso de la herramienta GeoGebra en la enseñanza-aprendizaje de la Matemática, a fin de proponer una guía para la formulación de un plan de estrategia didáctica, este se fundamentó desde el enfoque positivista y se desarrolló con base en la metodología cuantitativa. Atendiendo a esto, el diseño de investigación se define como de campo, no experimental, transeccional, dado que las variables de estudio se observaron tal cual como se dan en su contexto real, en un momento único y sin manipulación deliberativa de las mismas (Palella y Martins, 2015). Asimismo, la investigación se desarrollo a nivel proyectivo; pues se generó una guía para la formulación de un plan de estrategia didáctica como propuesta y con base en los resultados obtenidos que describen y explican el uso de la herramienta GeoGebra en la enseñanza-aprendizaje de la Matemática (Hurtado, 2000 en Palella y Martins, 2015).

La población estuvo conformada por 84 docentes del área de matemática de la ciudad de Azogues. Con respecto a la muestra, está se constituyo con 71 docentes escogidos mediante la técnica de muestreo aleatorio estratificado, con un $95 \%$ de nivel de confianza y un $4.75 \%$ de margen de error. La recolección de los datos en la realidad se dio mediante la técnica de la encuesta, de manera online y orientada a conocer y describir el uso recursos tecnológicos y GeoGebra en el aula de clases; para ello se utilizó un cuestionario de tipo escala Likert, cuya eficacia en términos de confiabilidad del instrumento se dio mediante la prueba de coeficiente de Alfa de Cronbach, obteniendo una fiabilidad de 0.890 (Palella y Martins, 2015).

Para cerrar, el análisis de la información recopilada se dio con sustento en la estadística descriptiva y la prueba de Chi-cuadrado de Pearson; asimismo, para la estructuración de los resultados de la investigación el software IBM SPSS Statistics 22.0 (Palella y Martins, 2015). 
Jenny Fernanda Alvarez-Matute; Darwin Gabriel Garcia-Herrera; Cristián Andrés Erazo-Álvarez; Juan Carlos Erazo-Álvarez

\section{RESULTADOS}

\section{Tabla 1.}

Frecuencia de docentes que utilizan material convencional (libros, material impreso, fotocopias, pizarra) en la clase de Matemática.

\begin{tabular}{llcccc} 
& & Frecuencia & Porcentaje & Porcentaje válido & $\begin{array}{c}\text { Porcentaje } \\
\text { acumulado }\end{array}$ \\
\hline \multirow{3}{*}{ Válido } & Ocasionalmente & 5 & 7,0 & 7,0 & 7,0 \\
& Casi siempre & 26 & 36,6 & 36,6 & 43,7 \\
& Siempre & 40 & 56,3 & 56,3 & 100,0 \\
& Total & 71 & 100,0 & 100,0 &
\end{tabular}

Fuente: Elaboración propia (2020).

Los resultados que se presentan en la tabla muestran que el $92.9 \%$ de los docentes del área de Matemática encuestados, en sus clases siempre o casi siempre utilizan material convencional como libros, material impreso, fotocopias, pizarra. En este sentido, se puede decir que a pesar de estar en pleno siglo XXI y rodeados de los avances tecnológicos, muchos docentes del área de Matemática permanecen coligados a la enseñanza tradicional y atados a los textos, los cuadernos y los lápices para impartir los contenidos de dicha asignatura, y al parecer no hacen uso de alternativas de enseñanza enfocada en las TIC, que en la actualidad están presente en el día a día de los estudiantes. 
Jenny Fernanda Alvarez-Matute; Darwin Gabriel Garcia-Herrera; Cristián Andrés Erazo-Álvarez; Juan Carlos Erazo-Álvarez

Tabla 2.

Frecuencia de docentes que utilizan software educativo.

\begin{tabular}{llcccc} 
& Frecuencia & Porcentaje & Porcentaje válido & $\begin{array}{c}\text { Porcentaje } \\
\text { acumulado }\end{array}$ \\
\hline \multirow{4}{*}{ Válido } & Nunca & 8 & 11,3 & 11,3 & 11,3 \\
& Casi nunca & 11 & 15,5 & 15,5 & 26,8 \\
& Ocasionalmente & 32 & 45,1 & 45,1 & 71,8 \\
& Casi siempre & 12 & 16,9 & 16,9 & 88,7 \\
& Siempre & 8 & 11,3 & 11,3 & 100,0 \\
& Total & 71 & 100,0 & 100,0 & \\
\hline
\end{tabular}

Fuente: Elaboración propia (2020).

Los resultados de la tabla 2 revelan que el $71,8 \%$ de los docentes encuestados ocasionalmente, casi nunca y/o nunca utilizan algún tipo de software educativo para impartir su cátedra de Matemática. Lo que quiere decir que a pesar de existir un sin número de aplicaciones o programas informáticos online y offline destinados al área de conocimiento de Matemática para que las clases sean más activas y participativas la frecuencia con que los docentes los utilizan es significativamente baja; evidenciándose con ello que pareciera no existir un acercamiento a muchos de estos recursos por la mayoría de los docentes, cuya causa puede tratarse por desconocimiento de las mismos, falta de capacitación para su manejo, recursos insuficientes, entre otras. 
Jenny Fernanda Alvarez-Matute; Darwin Gabriel Garcia-Herrera; Cristián Andrés Erazo-Álvarez; Juan Carlos Erazo-Álvarez

\section{Tabla 3.}

Frecuencia de uso de GeoGebra por parte de los docentes.

\begin{tabular}{llcccc} 
& Frecuencia & Porcentaje & Porcentaje válido & $\begin{array}{c}\text { Porcentaje } \\
\text { acumulado }\end{array}$ \\
\hline \multirow{3}{*}{ Válido } & Nunca & 20 & 28,2 & 28,2 & 28,2 \\
& Casi nunca & 9 & 12,7 & 12,7 & 40,8 \\
& Ocasionalmente & 24 & 33,8 & 33,8 & 74,6 \\
& Casi siempre & 11 & 15,5 & 15,5 & 90,1 \\
& Siempre & 7 & 9,9 & 9,9 & 100,0 \\
& Total & 71 & 100,0 & 100,0 & \\
\hline
\end{tabular}

Fuente: Elaboración propia (2020).

Con respecto a los resultados de la tabla 3 exponen que el $74.6 \%$ de los docentes encuestados ocasionalmente, casi nunca y/o nunca utilizan el programa informático GeoGebra para impartir sus conocimientos matemáticos, cifra alta considerando los avances tecnológicos que existen en esta época. De ahí que sería muy importante que los docentes conozcan los beneficios que GeoGebra ofrece para que las clases de Matemática no se tornen abstractas y aburridas, tal es el caso cuando se explican $u$ ejercicios en la pizarra y el estudiante resuelve los demás de manera mecánica. Pues el uso de GeoGebra en el aula permite una mejor comprensión de los contenidos de la asignatura haciéndola cada vez más interesante.

Para una descripcion mas profunda del uso de la herramienta GeoGebra en la enseñanza-aprendizaje de la Matemática se ha realizado la prueba Chi cuadrado de Pearson, contrastando las siguientes hipótesis: $\mathrm{H}_{1}$ (Hipótesis alternativa) el uso del software educativo está relacionado significativamente con el uso de GeoGebra, con la hipótesis $\mathrm{H}_{0}$ (hipótesis nula) el uso del software educativo no está relacionado significativamente con el uso de GeoGebra. 
EPISTEME KOINONIA

Revista Electrónica de Ciencias de la Educación, Humanidades, Artes y Bellas Artes

Año III. Vol III. N6. Julio - Diciembre 2020

Hecho el depósito de Ley: FA2018000022

ISSN: 2665-0282

FUNDACIÓN KOINONIA (F.K).

Santa Ana de Coro, Venezuela

Jenny Fernanda Alvarez-Matute; Darwin Gabriel Garcia-Herrera; Cristián Andrés Erazo-Álvarez; Juan Carlos Erazo-Álvarez

\section{Tabla 4.}

Tabulación cruzada: Frecuencia con la que los docentes utilizan el software educativo Frecuencia con la que los docentes utilizan el GeoGebra.

\begin{tabular}{|c|c|c|c|c|c|c|c|}
\hline & & \multicolumn{5}{|c|}{ Frecuencia con que los docentes utilizan el GeoGebra } & \multirow[b]{2}{*}{ Total } \\
\hline & & Nunca & $\begin{array}{c}\text { Casi } \\
\text { nunca }\end{array}$ & Ocasionalmente & $\begin{array}{c}\text { Casi } \\
\text { siempre }\end{array}$ & Siempre & \\
\hline Frecuencia & Nunca & 7 & 1 & 0 & 0 & 0 & 8 \\
\hline con la que los & Casi nunca & 6 & 3 & 2 & 0 & 0 & 11 \\
\hline docentes utilizan & Ocasionalmente & 6 & 3 & 19 & 4 & 0 & 32 \\
\hline el software & Casi siempre & 1 & 0 & 2 & 7 & 2 & 12 \\
\hline educativo & Siempre & 0 & 2 & 1 & 0 & 5 & 8 \\
\hline Total & & 20 & 9 & 24 & 11 & 7 & 71 \\
\hline
\end{tabular}

Fuente: Elaboración propia (2020)

Tabla 5.

Prueba de Chi-cuadrado

\begin{tabular}{lccc} 
& Valor & gl & Sig. asintótica (2 caras) \\
\hline Chi-cuadrado de Pearson & $80,721^{\mathrm{a}}$ & 16 & 0,000 \\
Razón de verosimilitud & 73,472 & 16 & 0,000 \\
Asociación lineal por lineal & 33,209 & 1 & 0,000 \\
N de casos válidos & 71 & &
\end{tabular}

a. 23 casillas $(92,0 \%)$ han esperado un recuento menor que 5 . El recuento mínimo esperado es ,79.

Fuente: Elaboración propia (2020). 
Jenny Fernanda Alvarez-Matute; Darwin Gabriel Garcia-Herrera; Cristián Andrés Erazo-Álvarez; Juan Carlos Erazo-Álvarez

Los resultados obtenidos en la tabla 4, pruebas de Chi-cuadrado, muestra que el valor de significación asintótica ( 2 caras) es de $0,00<0,05$; dando lugar a la hipótesis alternativa $\left(\mathrm{H}_{1}\right)$ descartándose la hipótesis nula $\left(\mathrm{H}_{0}\right)$, es decir el uso de software educativo está relacionado significativamente con el uso de GeoGebra.

En cuanto a la percepción de los docentes sobre el tipo de pensamiento matemático que desarrolla GeoGebra en los estudiantes se ha obtenido opiniones diversas. No obstante, resalta significativamente que mientras el $49.3 \%$ expresa que el GeoGebra desarrolla un tipo de pensamiento numérico, el $28,2 \%$ de los docentes encuestados no tienen conocimiento de la existencia del software.

Cabe agregar que de acuerdo a los resultados obtenidos del estudio realizado con docentes de la ciudad de Azogues sobre el uso del software educativo GeoGebra en las clases de Matemática, se puede decir que se ha verificado que no se da un acercamiento significativo a este tipo de herramienta, a pesar de que otorga múltiples ventajas para el desarrollo del proceso de enseñanza-aprendizaje en el sentido de que motiva hacia el aprendizaje y la construcción del conocimiento matemático de forma innovadora; idea ultima que es corroborada por (Zulnaidi \& Zamri, 2017) cuando expresan que los estudiantes que utilizan GeoGebra para aprender Matemática obtienen un mayor conocimiento conceptual y procedimental en relación con aquellos que lo hacen de forma convencional y/o tradicional. Para cerrar y considerando los resultados obtenidos con el estudio, a continuación, se propone una guia metodológica para la formulación de un plan de estrategia didáctica para el uso de la herramienta GeoGebra como alternativa en el proceso de enseñanza-aprendizaje de la Matemática. 
Jenny Fernanda Alvarez-Matute; Darwin Gabriel Garcia-Herrera; Cristián Andrés Erazo-Álvarez; Juan Carlos Erazo-Álvarez

\section{PROPUESTA}

Esta propuesta surge a razón de los resultados obtenidos en la investigación y con la finalidad de proponer a los docentes del Área de Matemática una guía metodológica para la formulación de un plan que implique el uso alternativo de GeoGebra como estrategia didáctica en el proceso de enseñanza-aprendizaje de dicha asignatura. Por consiguiente, se incorpora el modelo ADDIE como estructura que guia metodologicamente el desarrollo del plan de estrategia didáctica y que se constituye con el desarrollo de cinco (5) fases descritas asi: análisis, diseño, desarrollo, implementación y evaluación.

Figura 1. Modelo ADDIE.

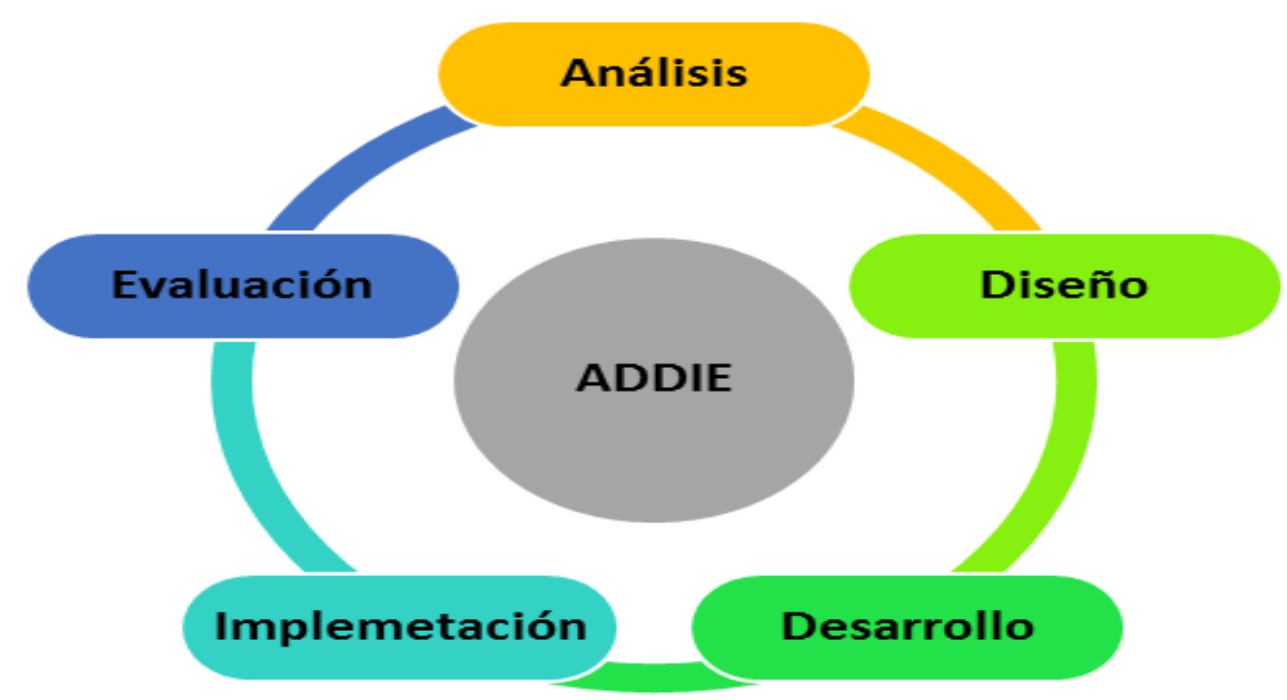

Fuente: Elaboración propia (2020). 
Jenny Fernanda Alvarez-Matute; Darwin Gabriel Garcia-Herrera; Cristián Andrés Erazo-Álvarez; Juan Carlos Erazo-Álvarez

1. Análisis: En esta fase el docente debe tener en cuenta el grupo de estudiantes con quienes trabajara para el abordaje de los conocimientos matemáticos. Este análisis debe estar orientado desde aspectos como:

$\checkmark$ Los conocimientos previos que tienen y que deberían tener los estudiantes sobre matemática e informática.

$\checkmark$ Las personas con necesidades educativas especiales.

$\checkmark$ El entorno donde va a enseñar que puede ser un laboratorio de computación o en una sala con dispositivos propios de los estudiantes.

$\checkmark$ Los contenidos matemáticos con los que se van a trabajar.

2. Diseño: En la fase de diseño el docente debe formular los objetivos y los contenidos de aprendizaje, además de organizar los diferentes recursos y materiales que va a utilizar en cada una de las actividades; en este sentido, se deben incluir los contenidos de aprendizaje en diferentes formas ya sea texto, audio y video compatibles con el software GeoGebra que será la herramienta principal para llevar a cabo el proceso.

3. Desarrollo: En esta fase, los contenidos, recursos y materiales necesarios para desarrollar la asignatura, se enlazan y/o vinculan con la herramienta GeoGebra, teniendo claro el docente la orientación que pretende brindar con el uso de la aplicación y que pueden ser: de análisis, representación, interpretación, simulación, animación, entre otras del tema establecido.

4. Implementación: Esta fase implica el despliegue del proceso formativo en sí; es decir, involucra el desarrollo de los contenidos matemáticos vinculados a la herramienta GeoGebra. Para ello, previamente se ha considerando la capacitación previa del docente en cuanto al manejo de GeoGebra para ofrecer un acompañamiento más práctico a los estudiantes durante todo el proceso.

5. Evaluación: Finalmente, en esta fase de evaluación se determina si se han 
Jenny Fernanda Alvarez-Matute; Darwin Gabriel Garcia-Herrera; Cristián Andrés Erazo-Álvarez; Juan Carlos Erazo-Álvarez

cumplido o no lo planificado. Para ello, se debe considerar los dos tipos de evaluación: la formativa que se lleva en el transcurso del proceso de aprendizaje y la sumativa al final del mismo. La evaluación se despliega con los recursos para el aula que contiene el software GeoGebra, ya que la aplicación ofrece un sin número de modelos online que son colaboraciones de muchos usuarios y también hay la posibilidad de que el docente genere los suyos propios. Cabe decir, que los resultados de la evaluación han de servir para conocer los aspectos a mejorar en relación a las fases anteriores del modelo.

\section{CONCLUSIONES}

Los resultados de la investigación muestran que existe un número considerable de docentes en el área de Matemática que utilizan formas convencionales y/o tradicionales para enseñar y no están familiarizados con el uso de software o programas educativos como GeoGebra para impartir sus conocimientos, por lo que es muy importante promover el uso de estos recursos para innovar en las aulas de clase. GeoGebra es una herramienta educativa que se basa en la representación simbólica y gráfica de los diferentes contenidos matemáticos para un aprendizaje más activo y significativo. Es decir, con esta herramienta los estudiantes pueden comprender de manera clara y oportuna lo conceptos y procedimientos al permitirles realizar demostraciones, simulaciones y representaciones, como complemento para cada momento del aprendizaje de la Matematica..

El modelo ADDIE propuesto para la implementación del uso de GeoGebra en el aula permite al docente identificar los aspectos positivos y negativos en cada fase del ciclo metodológico, para mejorar y renovar sus actividades didacticas y así obtener una mejor participación estudiantil. En concreto, se puede decir que el software educativo GeoGebra como herramienta tecnológica educativa ofrece muchas ventajas en lo que respecta a la didáctica de la Matemática; por consiguiente, es determinación de los 
Jenny Fernanda Alvarez-Matute; Darwin Gabriel Garcia-Herrera; Cristián Andrés Erazo-Álvarez; Juan Carlos Erazo-Álvarez

docentes aprovechar estos beneficios para cambiar la forma de impartir su asignatura en procura que sus estudiantes sean más activos y participativos.

\section{FINANCIAMIENTO}

No monetario.

\section{AGRADECIMIENTOS}

A los docentes del área de matemática de la ciudad de Azogues.

\section{REFERENCIAS}

Arévalo-Duarte, M. A., \& Gamboa-Suárez, A. A. (2015). TIC en el curriculo de matemáticas. Una orientación desde el marco de las politicas y proyectos educativos. [ICT in the mathematics curriculum. An orientation from the framework of educational policies and projects1]. Revista Interamericana de Investigación, Educación y Pedagogía, 8(1), 169-187.

Barahona-Avecilla, F., Barrera-Cárdenas, O., Vaca-Barahona, B., \& Hidalgo-Ponce, B. (2015). GeoGebra para la enseñanza de la matemática y su incidencia en el rendimiento académico estudiantil. [GeoGebra for the teaching of mathematics and its impact on student academic performance]. Revista Tecnológica-ESPOL, 28(5), 121-132.

Carrillo de Albornoz-Torres, A. (2019). Materiales y recursos para aprovechar lo que ofrece la comunidad GeoGebra. [Materials and resources to take advantage of what the GeoGebra community offers]. Memorias de la I Jornada Ecuatoriana de GeoGebra, 1, 51-60. Recuperado de https://n9.cl/m6yur

Cordero-Naspud, E. I., Erazo-Álvarez, J. C., Narváez-Zurita, C. I., \& Cordero-Guzmán, D. M. (2020). Soluciones corporativas de inteligencia de negocios en las pequeñas y medianas empresas. [Corporate business intelligence solutions in small and medium enterprises]. Revista Arbitrada Interdisciplinaria Koinonía, 10(5); 483-513. http://dx.doi.org/10.35381/r.k.v5i10.703 
Jenny Fernanda Alvarez-Matute; Darwin Gabriel Garcia-Herrera; Cristián Andrés Erazo-Álvarez; Juan Carlos Erazo-Álvarez

Erazo-Álvarez, J. C., \& Narváez-Zurita, C. I. (2020). Medición y gestión del capital intelectual en la industria del cuero - calzado en Ecuador. [Measurement and management of intellectual capital in the leather industry - footwear in Ecuador]. Revista Arbitrada Interdisciplinaria Koinonía, 9(5); 437-467. http://dx.doi.org/10.35381/r.k.v5i9.662

Fernández, I., Riveros, V., \& Montiel, G. (2017). Software educativo y las funciones matemáticas. Una estrategia de apropiación. [Educational software and mathematical functions. An appropriation strategy]. Omnia, 23(1), 9-19.

Guerrero-Garcés, L., Villa-Escudero, I., Martínez-Muñoz, O., \& Hernández-Allauca, A. (2018). El uso del software GeoGebra en la resolución de sistemas de inecuaciones. [The use of GeoGebra software in solving inequality systems]. Revista Atlante: Cuadernos de Educación y Desarrollo. Recuperado de https://n9.cl/xzud

Instituto Nacional de Evaluación Educativa (2018). La educación en Ecuador: logros alcanzados y nuevos desafíos. Resultados educativos 2017/2018. [Education in Ecuador: achievements and new challenges. Educational result 2017/2018]. Recuperado de https://n9.cl/5yig

Ministerio de Educación del Ecuador. (2016). Currículo de los niveles de educación obligatoria. Recuperado de https://n9.cl/mnli

Orrantia, J. (2006). Dificultades en el aprendizaje de las matemáticas: una perspectiva evolutiva. [Difficulties in learning mathematics: an evolutionary perspective]. Revista Psicopedagogia, 23(71), 158-180.

Palella, S. \& Martins F. (2015). Metodologia de la investigacion cuantitativa. [Methodology of quantitative research]. Venezuela. FEDEUPEL

Pari-Condori, A. (2019). El impacto de GeoGebra en el desarrollo profesional del profesorado de matemáticas. [GeoGebra's impact on the professional development of mathematics teachers]. Memorias de la I Jornada Ecuatoriana de GeoGebra, 1, 23-36. Recuperado de https://n9.cl/5bbt

Rodríguez, D., Erazo, J., \& Narváez, C. (2019). Técnicas cuantitativas de investigación de mercados aplicadas al consumo de carne en la generación millennial de la ciudad de Cuenca (Ecuador) [Quantitative market research techniques applied to meat consumption in the millennial generation of the city of Cuenca (Ecuador)] Revista Espacios, 40(32); 20. 
EPISTEME KOINONIA

Revista Electrónica de Ciencias de la Educación, Humanidades, Artes y Bellas Artes

Año III. Vol III. N6. Julio - Diciembre 2020

Hecho el depósito de Ley: FA2018000022

ISSN: 2665-0282

FUNDACIÓN KOINONIA (F.K).

Santa Ana de Coro, Venezuela

Jenny Fernanda Alvarez-Matute; Darwin Gabriel Garcia-Herrera; Cristián Andrés Erazo-Álvarez; Juan Carlos Erazo-Álvarez

Rodríguez-Uribe, L. A. (2017). GeoGebra como recurso educativo para la Enseñanza de las matemáticas en educación superior. [GeoGebra as an educational resource for Teaching mathematics in higher education]. Universidad Militar Nueva Granada. Recuperado de https://n9.cl/zn71

Salas-Rueda, R. A. (2018). Uso del servicio en la nube GeoGebra durante el proceso enseñanza-aprendizaje sobre las matemáticas. [Use of the GeoGebra cloud service during the teaching-learning process on mathematics]. RIDE Revista Iberoamericana para la Investigación y el Desarrollo Educativo, 8(16), 23-52.

Saltos-Cedeño, A., Vallejo-Valdivieso, P., \& Moya-Martínez, M. (2020). Innovación en educación matemática de básica superior durante el confinamiento por COVID19. [Innovation in higher basic mathematics education during confinement by COVID-19]. EPISTEME KOINONIA, $\quad 3(5), \quad 142-161$. http://dx.doi.org/10.35381/e.k.v3i5.723

Tamayo-Martínez, E. D. (2013). Implicaciones didácticas de GeoGebra sobre el aprendizaje significativo de los tipos de funciones en estudiantes de secundaria [Didactic implications of GeoGebra on the significant learning of the types of functions in secondary school students]. Apertura, 5(2), 58-69.

Vargas-Vargas, G., \& Gamboa-Araya, R. (2013). La enseñanza del teorema de Pitágoras: una experiencia en el aula con el uso del GeoGebra, según el modelo de van hiele [Teaching the Pythagorean theorem: an experience in the classroom with the use of GeoGebra, according to the van ice model]. Uniciencia, 27(1), 95118.

Zulnaidi, H., \& Zamri, S. (2017). The effectiveness of the GeoGebra software: The intermediary role of procedural knowledge on students' conceptual cnowledge and their achievement in Mathematics [La efectividad del software GeoGebra: El rol intermediario del conocimiento procesal en]. Eurasia Journal of Mathematics, Science and Technology Education, 13(6), 2155-2180.

C2020 por el autor. Este artículo es de acceso abierto y distribuido según los términos y condiciones de la licencia Creative Commons Atribución-NoComercial-Compartirlgual 4.0 Internacional (CC BY-NC-SA 4.0)

(https://creativecommons.org/licenses/by-nc-sa/4.0/). 